\title{
Transformation of polycrystalline tungsten to monocrystalline tungsten W(100) and its potential application in Schottky emitters
}

\author{
A. K. Dokania ${ }^{a}$ \\ Faculty of Applied Science, Delft University of Technology, Lorentzweg 1, 2628 CJ Delft, The Netherlands \\ Ruud Hendrikx \\ Department of Materials Science and Engineering, Delft University of Technology, \\ Faculty of 3mE Mekelweg 2, 2628 CJ Delft, The Netherlands \\ P. Kruit \\ Faculty of Applied Science, Delft University of Technology, Lorentzweg 1, 2628 CJ Delft, The Netherlands
}

(Received 23 June 2008; accepted 1 December 2008; published 12 January 2009)

\begin{abstract}
The electron sources in electron microscopes and electron lithography machines often consist of small diameter W(100) wires, etched to form a sharp tip. The electron emission is facilitated by the Schottky effect, thus the name Schottky emitter. The authors are investigating the feasibility of arrays of such electron emitters for the use in multibeam lithography. This, however, would require large pieces of $\mathrm{W}(100)$ which are not easily available. The concept of dc Joule heating for the crystallographic transformation is utilized to convert polycrystalline tungsten to $\mathrm{W}(100)$ for the fabrication of the Schottky emitters. A miniature Schottky emitter fabricated by wire electrical discharge machining was heated by $\mathrm{dc}$ and the crystallographic orientation was subsequently determined by electron diffraction. The x-ray diffraction measurement on another sample of tungsten filament, heated by similar method, confirms the above results. The concept will be extended for the fabrication of a prototype multibeam source for parallel electron beam lithography. () 2009 American Vacuum Society. [DOI: 10.1116/1.3058713]
\end{abstract}

\section{INTRODUCTION}

The Schottky emitter is known for its high brightness and high current stability and would be the ideal electron source for a parallel electron beam lithography system. A single tip, however, does not provide sufficient current. We are thus investigating the possibility of creating an array of Schottky emitters at a pitch of $1.5 \mathrm{~mm}$ for such a system. The current method of preparation of Schottky emitters involve the electrochemical etching of W(100) wire. However, W(100) is not easily available in all desired dimensions needed for the fabrication of arrays of tips. In this paper we explore the possibility of using polycrystalline tungsten for the fabrication of a Schottky emitter array.

In a paper by Okuyama, ${ }^{1}$ the author described the transformation of polycrystalline tungsten to $\mathrm{W}(100)$. However, the process involved a complex heating process of carburizing and decarburizing the tungsten tip. There were attempts to make monocrystalline tungsten ribbon of (113) from polycrystalline ribbon by strain annealing, which, however, did not give control over the surface plane normal to the axis. ${ }^{2,3}$ For polycrystalline tungsten, normally after heating, the preferred orientation of grain formation is (110) owing to its low surface free energy. The orientation of the crystal structure during heating is also greatly influenced by the rolling or drawing direction. The crystallographic transformation of polycrystalline tungsten to $\mathrm{W}(100)$ by dc Joule heating is observed in welding arc electrodes. ${ }^{4,5}$ However, this phenomenon was never utilized for the fabrication of electron

\footnotetext{
a)Tel.: +31-152781516; electronic mail: a.k.dokania@tudelft.nl
}

emitters. In this paper an attempt is made to utilize this phenomenon for the fabrication of Schottky emitters.

\section{EXPERIMENTAL METHOD}

We have reported ${ }^{6}$ the fabrication of Schottky emitters of $1 \mathrm{~mm}$ diameter of pure polycrystalline tungsten by wire electrical discharge machining (WEDM) also known as spark erosion, where the schematic design of which is shown in Fig. 1. After the dc Joule heating at $1500-1550{ }^{\circ} \mathrm{C}$ for several days, the tips were observed in a scanning electron microscope (SEM). Figure 2 shows several tips fabricated by WEDM, after dc Joule heating, depicting parallel grain structures. The similar pattern of the grain structure led to further investigation for the crystallographic orientation of the grains. X-ray diffraction technique could not be used to determine the crystal orientation of the tips as $\mathrm{x}$-ray beam could not be confined to such a small area. The tip shown in the top-left in Fig. 2 is investigated by electron diffraction to know the exact crystallographic orientation of the grains. The sample was thinned down by focused ion beam (FIB) to make it electron transparent, shown in Fig. 3. At first, a layer of platinum was deposited as a protective layer which facilitates milling at $5000 \mathrm{pA}$ without damaging the sample [Fig. $3(b)]$, subsequently the ion current was lowered in steps to few hundreds for the final milling to approximately $150 \mathrm{~nm}$ [Fig. 3(c)]. The electron diffraction experiment was performed on a Philips CM30UT electron microscope at $300 \mathrm{kV}$.

To further confirm the above results, another characterization method, x-ray diffraction (XRD) is used on another 


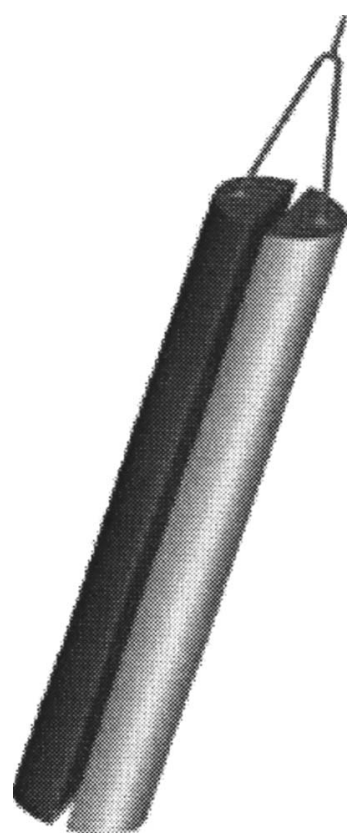

FIG. 1. Schematic of $1 \mathrm{~mm}$ diameter Schottky emitter fabricated by WEDM.

tungsten filament with different thermal history than above, and samples of $1 \mathrm{~mm}$ length were cut from the different parts of the filament/tip [Fig. 5(a)]. Each sample was glued with the filament axis parallel to the surface on a substrate consisting of a Si single crystal wafer with orientation 510. Diffractometer scans were made with a Bruker-AXS D5005 equipped with an incident-beam $\mathrm{Cu} K \alpha 1$ monochromator and a Braun position sensitive detector. Texture measurements were made with a Bruker-AXS D8 Discover diffractometer equipped with an Eulerian cradle and parallel beam optics using (filtered) Co $K \alpha$ radiation. Pole figures of the

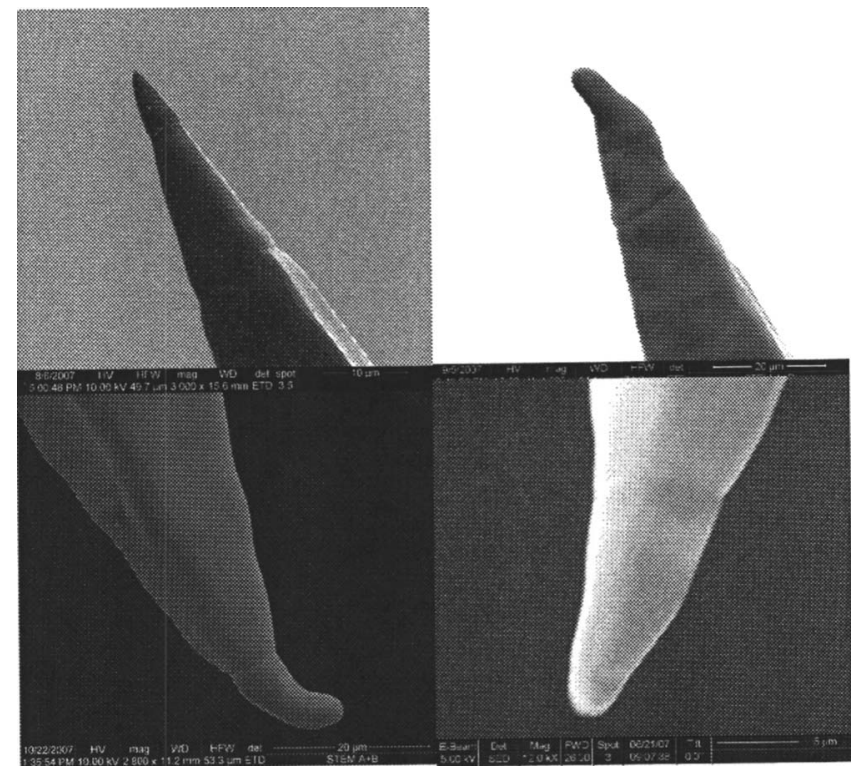

FIG. 2. Morphology of different tips made by WEDM after dc heating.

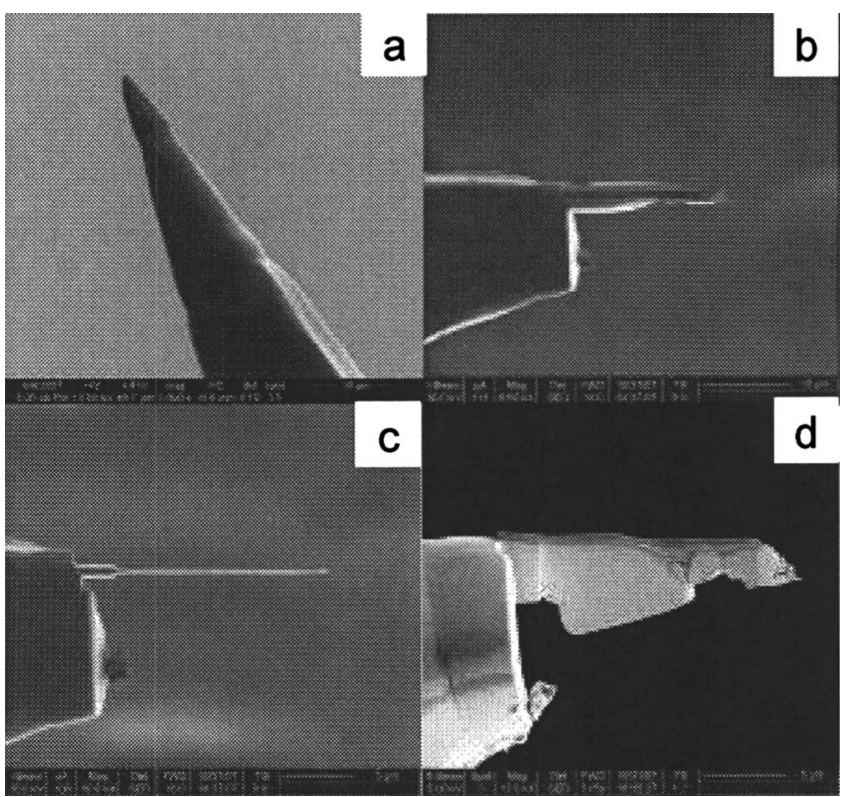

FIG. 3. FIB milling of the WEDM sample for electron diffraction experiment (a), tip (b) after first step of FIB milling at $5000 \mathrm{pA}$, (c) final FIB milled sample, and (d) the same sample rotated at $90^{\circ}$.

$\mathrm{W}\{110\}$ and $\mathrm{W}\{200\}$ reflections were obtained by rotating the sample (around the wafer normal) from 0-360 and tilting it from $0-70$ in steps of $2^{\circ}$.

\section{RESULTS AND DISCUSSION}

The electron diffraction results are shown in Fig. 4. The zone axis for the diffraction pattern is [001]. The basic lattice structure reflections of (200) and (020) are indicated by arrows, which denote the reciprocal lattice vectors. In case of tungsten, because of symmetry, diffraction from $(h, k, l, h$ $+k+l=$ odd) planes are forbidden and only diffraction from

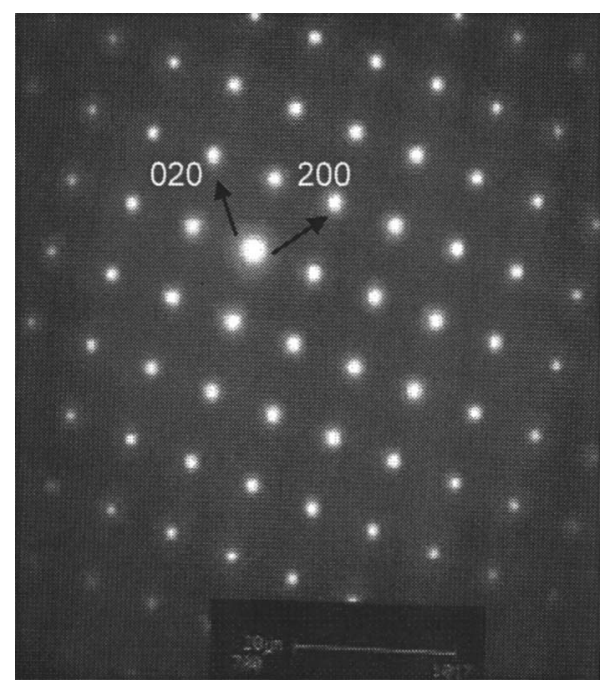

FIG. 4. Electron diffraction pattern from the sample in Fig. 3, confirming $\mathrm{W}(100)$ orientation. 


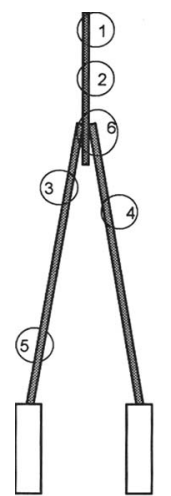

(a)

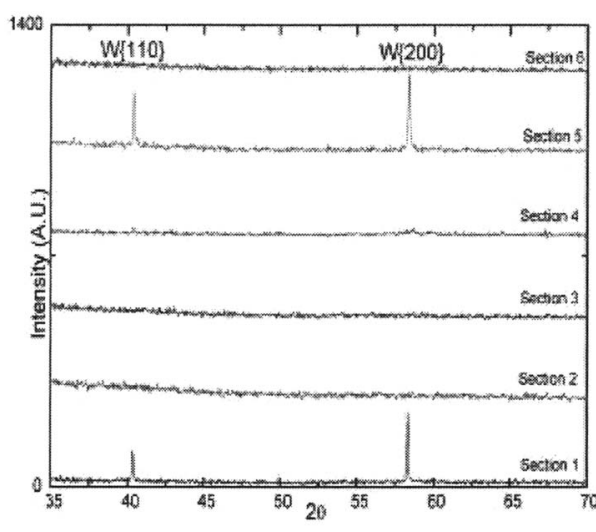

(b)
FIG. 5. (a) Parts of the sample taken for XRD measurement. (b) XRD pattern from different parts of the filament shown in (a).

$(h, k, l, h+k+l=$ even) planes are observed, which is in accordance to the diffraction rule for a bcc material such as tungsten.

The diffractometer scans from the various portions of the heated tip and filament are shown in Fig. 5. For the regions 1 and 5, both the $\mathrm{W}\{110\}$ and $\mathrm{W}\{100\}$ reflections are present, indicating that more than one type of planes is oriented parallel to the surface. However, the patterns from regions 2, 3, 4 , and 6 do not show any diffraction peak, indicating the absence of such planes parallel to the surface. This may suggest a strong directional crystal growth, which could be due to the fact that the temperature at those regions is the highest. To further investigate the crystal orientation from the heated portion of the filament, samples 3 and 4 were investigated by XRD pole figure measurements. The pole figures from sample 3 (not shown) did present some intensity maxima, but a distinct orientation could not be derived from them.

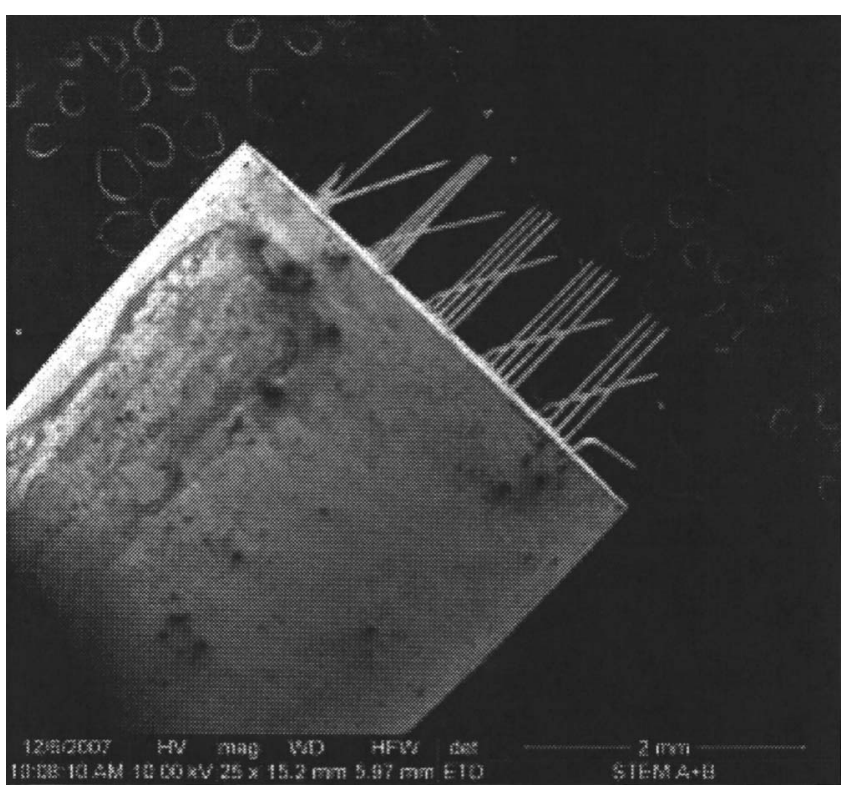

FIG. 7. Tip array made from polycrystalline tungsten by WEDM technique.

Figures 6(a) and 6(b) show the pole figures from sample 4 obtained for the $\mathrm{W}\{110\}$ and $\mathrm{W}\{200\}$, respectively. The relatively broad intensity maxima originate from the $\mathrm{W}$ filament, while the other sharp maxima originate from the Si 510 wafer. The positions of the broad intensity maxima and the combination of both patterns indicate a single crystal orientation with the (100) plane almost parallel to the filament axis with a deviation of about $10^{\circ}$. The above measurement confirms that the filament in the hottest part transform to $\mathrm{W}$ (100) single crystal. The temperature distribution across such filament configuration can be found in Ref. 7. Apparently the

$$
\text { : }
$$

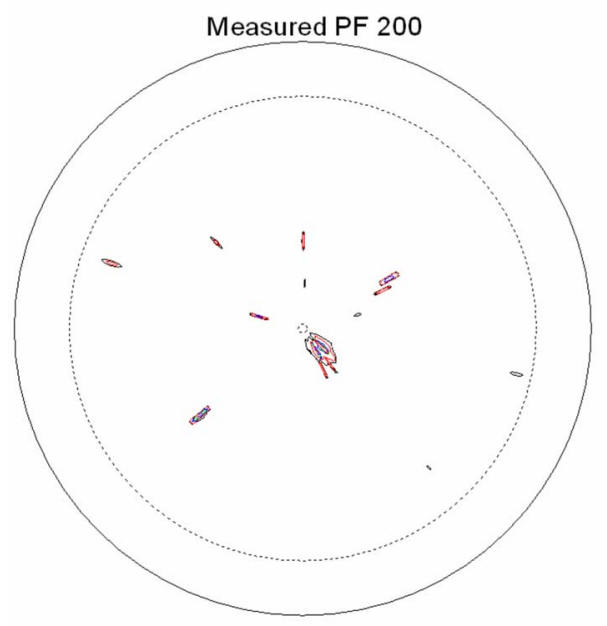

$:=$

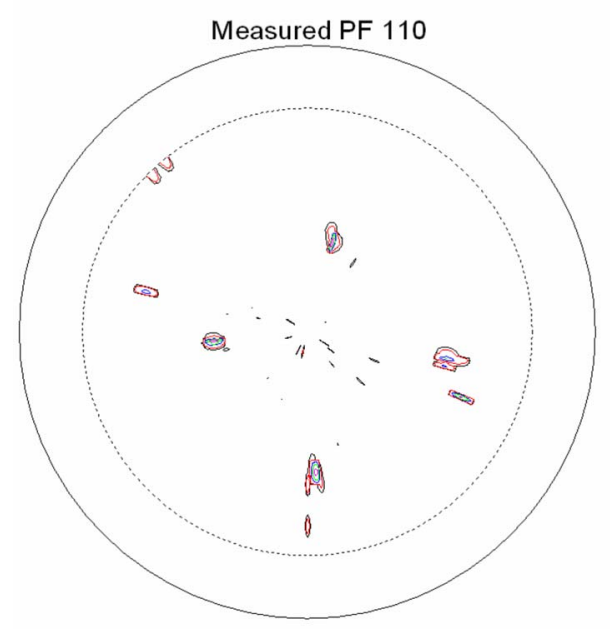

FIG. 6. Pole figures from sample 4 obtained for (a) $\mathrm{W}\{200\}$ and (b) $\mathrm{W}\{110\}$. 

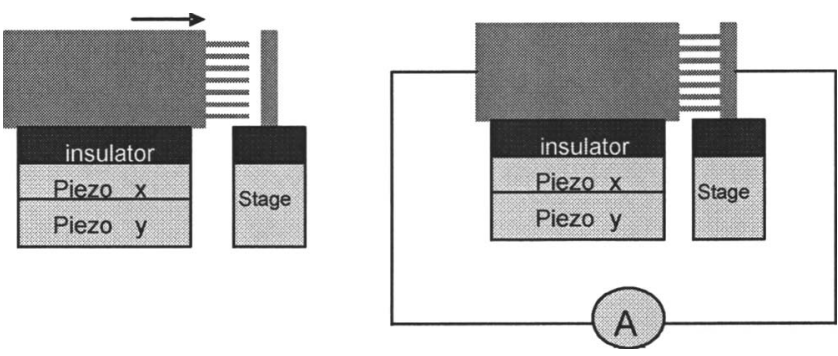

FIG. 8. Schematic diagram of dc Joule heating for the sample shown in Fig. 7 .

transformation in the "cooler" parts was not complete, which could be due to the kinetics of the transformation, described below.

Normally after heating, $\mathrm{W}(110)$ is formed owing to its low surface free energy. However, during dc Joule heating, because of anisotropy in conductivity at higher temperature, the current density vector induces recrystallization in a direction which has least resistance. ${ }^{4,5}$ For BCC metals such as tungsten it is the $\langle 100\rangle$ direction, and for fcc it is the $\langle 110\rangle$ direction along which minimum resistivity occurs. In our setup, the current does not actually flow through the tip, so we conclude that the transformed $\mathrm{W}(100)$ in the heating filament must have acted as a seed crystal for the recrystallization of the tip. The kinetics of the transformation given by Eq. (1) shown below,

$$
u=\nu \cdot d \cdot \exp \left(-\frac{U}{k \cdot T}\right) \cdot \frac{3 \Delta T}{T},
$$

where $\nu$ is the Debye frequency, $d$ is the width of the interface, $U$ is the activation energy for vacancy transportation at high temperatures, $k$ is the Boltzmann constant, and $\Delta T$ is the temperature difference between grains. The value of $\Delta T$ is dependent on the purity of the sample as impurities along the grain boundary can increase the width of the interface and the temperature difference between two adjacent grains. The above equation can provide a guideline for the temperature-time needed for the complete transformation to (100). By the similar concept of dc Joule heating, Greiner and Kruse $^{8}$ reported to obtain sharp STM tips in the (110) direction. This could be explained by the above equation. The wire by Greiner and $\mathrm{Kruse}^{8}$ was heated at $1700{ }^{\circ} \mathrm{C}$ only for $30 \mathrm{~min}$, which was not sufficient for the complete $\mathrm{W}(100)$ transformation.

We will extend the same concept of WEDM and crystallographic transformation for the fabrication of the multibeam source. An example of multitip fabrication by WEDM is shown in Fig. 7. The crystallographic transformation to $\mathrm{W}(100)$ will be done by dc Joule heating, where the schematic of which is shown in Fig. 8. It consists of a piezoelectric stage on which the tip array is placed which can be moved towards a counter electrode of tungsten to complete the circuit. The heating current for an individual tip is the current through the block divided by the number of tips touching the plate. The current for an individual tip is determined by the temperature needed for the transformation and the necessary current is set with the help of a thermal model. $^{7}$ The above experiment will be done in a SEM to monitor the tip-disk contact. The electron emission experiment of such a tip and fabrication of array of tips is underway.

\section{CONCLUSION}

The simple concept of dc Joule heating for the crystallographic transformation of polycrystalline tungsten to W(100) can be used for the fabrication of Schottky emitters. This phenomenon has been demonstrated in a polycrystalline emitter made by WEDM technique and normal polycrystalline wire. This technique along with the WEDM technique can be extended for the fabrication of a multibeam source required for parallel electron beam lithography systems. The above method facilitates the use of polycrystalline tungsten of any shape and size.

\section{ACKNOWLEDGMENTS}

The authors would like to thank Marko Pelle from the Central workshop, TU Delft for the WEDM fabrication of the tips. The authors would also like to thank and acknowledge Alexandro Grabulov and Vikas Kumar of NCHRM, TU Delft for their help in FIB milling and electron diffraction, respectively.

${ }^{1}$ F. Okuyama, Phys. Status Solidi A 55, 793 (1979).

${ }^{2}$ F. L. Reynolds, J. Chem. Phys. 39, 1107 (1963).

${ }^{3}$ F. L. Reynolds, Rev. Sci. Instrum. 37, 1730 (1966).

${ }^{4}$ V. F. Gordeev, A. V. Pustogarov, Ya. R. Kucherov, V. A. Sukhinin, and G. M. Kudinov, Phys. Chem. Mech. Surf. 4, 3358 (1987).

${ }^{5}$ V. F. Gordeev, A. V. Pustogarov, Ya. R. Kucherov, and A. P. Khalboshin, Phys. Chem. Mater. Treat. 17, 469 (1983).

${ }^{6}$ A. K. Dokania, M. Pelle, and P. Kruit, Microelectron. Eng. 85, 1031 (2008).

${ }^{7}$ A. K. Dokania, J. F. M. Velthuis, Y. Zhang, and P. Kruit, J. Vac. Sci. Technol. B 25, 504 (2007).

${ }^{8}$ M. Greiner and P. Kruse, Rev. Sci. Instrum. 78, 026104 (2007). 